\title{
Impact of a Care Bundle on the Incidence of Necrotizing Enterocolitis in the Neonatal Intensive Care Unit
}

Jerome P. Lee, MD, Winona K. Lee, MD, Chieko Kimata, PhD, MPH, MBA, Alyssa Honda, MD, Charles R. Macías Neal, MD, PhD

\section{Abstract:}

Objective: Evaluate the impact of a care bundle on the incidence of necrotizing enterocolitis (NEC) in the neonatal intensive care unit.

Study Design: Retrospective, single-center, population comparison of patients diagnosed with NEC before and after implementing a NEC care bundle utilizing standardized feeding protocol, donor milk program, transfusion protocol, early antibiotic protocol, and restricted indomethacin use.

Result: Incidence of NEC fell from 1.92 to $0.83 \%(P<0.0001)$. Incidence of NEC in the 23-27 weeks gestation group decreased from 14.21 to $6.09 \%(P=0.0009)$. In the $28-30$ weeks gestation group, NEC incidence decreased from 5.56 to $2.10 \%(P=0.0096)$. Significant reduction of recurrent NEC and transfusion-associated NEC was observed.

Conclusion: Implementation of a NEC care bundle reduced NEC incidence, with the greatest impact seen in the most vulnerable preterm and very preterm infants.

\section{Introduction}

Necrotizing enterocolitis (NEC) remains a devastating disease of the premature infant. When encountered in the neonatal intensive care unit (NICU), NEC carries a mortality rate of up to $44 \%$, with significant long-term morbidity in survivors.(1)The incidence of NEC is highly variable and is dependent on gestational age and birth weight. Preterm and very preterm infants are at the highest risk for NEC. In a recent review by Battersby et al., NEC occurred in $5-7 \%$ of infants born $<35$ weeks gestation and in $5-22 \%$ of preterm infants with birth weight $<1000$ grams.(2 )Incidence of recurrent NEC has been reported to be $6-10 \% .(3,4)$

NEC delays the establishment of full enteral nutrition, leads to poor growth, and increases the length of hospital stay with a concomitant increase in hospital costs. Providing optimal nutritional support to premature infants is vital to their survival, growth, and long-term neurodevelopmental outcomes. (5-7) Optimizing nutrition while minimizing the occurrence of NEC continues to be a major challenge in the NICU.

Following decades of basic research into the mechanism of this disease, the pathophysiology of NEC remains poorly understood. No single etiology has been shown to cause NEC, and no single preventative measure has been shown to eliminate NEC. As a result, various prevention strategies have been reported in attempts to limit its occurrence.(8-17) Of the prevention strategies studied, establishing a standardized feeding protocol and exclusive human milk feeds appears to have the greatest impact on decreasing the

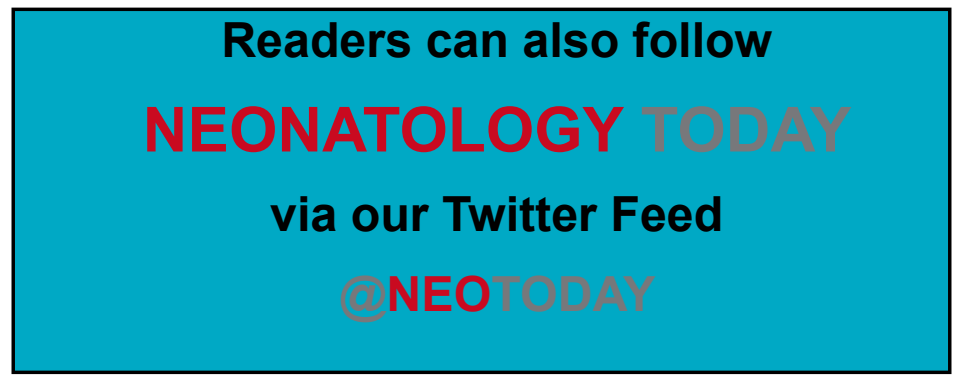

incidence of NEC in the premature infant.

To enhance the care of the premature infant and improve outcomes, quality improvement initiatives in the NICU are now becoming standard practice worldwide. Quality initiatives dealing with complex disease processes, such as NEC, have demonstrated that care bundles when properly initiated, improve patient care and ultimately patient outcomes. As defined by the Institute for Healthcare Improvement, care bundles are a group or set of evidence-based practice protocols that, when followed consistently together, can improve patient care processes and patient outcomes more than the individual protocols alone. $(18,19)$ In the NICU setting, care bundles have been shown to decrease the incidence of ventilator-associated pneumonia, central line-associated bloodstream infections, and nosocomial infections.(20-23 ) Recently, Talavera et al. reported a reduction in the incidence of NEC after the implementation of a quality improvement initiative that included early feedings with maternal breast milk (MBM), a feeding protocol, and limited use of ranitidine.(24)

\section{"A care bundle for NEC was developed and implemented for the level IIID NICU at Kapi"olani Medical Center for Women and Children in an effort to decrease NEC and its associated morbidity and mortality, particularly in the most fragile ELBW population. The purpose of this study was to retrospectively analyze the incidence, severity, and mortality rate of NEC in our NICU population before and after implementation of the NEC care bundle."}

A care bundle for NEC was developed and implemented for the level IIID NICU at Kapi'olani Medical Center for Women and Children in an effort to decrease NEC and its associated morbidity and mortality, particularly in the most fragile ELBW population. The purpose of this study was to retrospectively analyze the incidence, severity, and mortality rate of NEC in our NICU population before and after implementation of a NEC care bundle.

\section{Methods}

\section{NEC care bundle}

In November 2007, we implemented a NEC care bundle in the NICU, the elements of which included: 1) a standardized feeding protocol; 2) initiation of a donor milk program; 3) a transfusion protocol; 4) a protocol to limit early empiric antibiotics, and 5) restriction of the use of indomethacin.

\section{Standardized feeding protocol}

We developed a standardized feeding protocol that included all 
infants $<31$ weeks gestation and any infant $\geq 31$ weeks with any of the following risk factors: multiple gestation, congenital heart disease, perinatal asphyxia, intrauterine growth restriction, sepsis, and shock, gastroschisis, and polycythemia requiring partial exchange transfusion. The protocol included specific criteria for initiation and duration of trophic feedings, advancement of feedings, standard fortification practices, and use of parenteral nutrition.

We adopted a multidisciplinary approach with lactation consultants and nursing to establish early maternal breast milk feedings through maternal counseling and support. Education included information provided during the prenatal consult and an educational video on manual colostrum expression. An electric breast pump was provided to each mother. Lactation consultants were staffed to be available to the nursing mothers in the NICU 7 days a week.

\section{Donor breast milk program}

We also implemented a donor milk program that allowed infants to receive donated maternal breast milk (MBM) when the mother was unable to produce enough expressed MBM for her preterm infant. Donor breast milk (DBM) was obtained through a human milk bank licensed by the Human Milk Banking Association of North America. This assured supplementation of MBM feedings with a human milk alternative, reducing the use of preterm infant formula.

\section{Packed red cell transfusion protocol}

The packed red blood cell (PRBC) transfusion protocol included guidelines for PRBC transfusion based on infant age, need for respiratory support, and hemoglobin/hematocrit levels. Neonatologists agreed to not deviate from transfusion guidelines, limiting premature transfusion in VLBW infants while also not allowing hematocrits to become dangerously low. Transfusions were given in two $10 \mathrm{ml} / \mathrm{kg}$ aliquots, given 20-24 hours apart. Routine furosemide was not used. Feedings were decreased to trophic volumes during transfusion and advanced back to baseline volumes over 24-48 hours. Hemoglobin and hematocrit values were monitored weekly for infants up to 32 weeks post-conceptual age and every two weeks thereafter.

\section{Antibiotic protocol}

We modified our empiric antibiotic therapy practice by limiting empiric treatment in non-septic appearing infants to 48 hours. A time-out note is documented for any use of antibiotics beyond 48 hours if the initial baby blood culture is negative. Previously, empiric antibiotic therapy was given for a minimum of 72 hours and up to a week for infants with a negative blood culture.

"We had inconsistent practice among our providers with the use of indomethacin for intraventricular hemorrhage (IVH) prophylaxis, patent ductus arteriosus (PDA) prophylaxis and PDA treatment. We stopped the practice of using indomethacin for IVH and PDA prophylaxis and transitioned to ibuprofen as first-line medical therapy for the symptomatic PDA."

\section{Indomethacin protocol}

We had inconsistent practice among our providers with the use of indomethacin for intraventricular hemorrhage (IVH) prophylaxis, patent ductus arteriosus (PDA) prophylaxis and PDA treatment. We stopped the practice of using indomethacin for IVH and PDA prophylaxis and transitioned to ibuprofen as first-line medical therapy for the symptomatic PDA.

\section{Study Design}

After implementing our NEC care bundle, we conducted a 14 year retrospective population comparison study of two groups of patients diagnosed with NEC in the NICU at Kapi'olani Medical Center for Women and Children. The Pre-Bundle NEC group included infants born between 2004 and 2007 (4 year period). The Post-Bundle NEC group included infants born between 2008 and 2017 (10 year period). Infants were stratified into three categories,

Table 1 Incidence of NEC in Pre-Bundle and Post-Bundle Cohorts in the NICU

Pre-Bundle Frequency \%

54

26

23 to 27 week gestation

28 to 30 week gestation $\geq 31$ week gestation
14

14

9

13

27

14
$1.92 \%$

$14.21 \%$

$5.56 \%$

$0.59 \%$
Post-Bundle Frequency \% P-value
Recurrent NEC

Transfusion related NEC Surgical NEC

Death related to NEC

Abbreviations: NEC, necrotizing enterocolitis, NS, non-significant

\begin{tabular}{ccc}
70 & $0.83 \%$ & $<0.0001$ \\
33 & $6.09 \%$ & 0.0009 \\
14 & $2.10 \%$ & 0.0096 \\
23 & $0.32 \%$ & 0.084 \\
& & \\
1 & $1.43 \%$ & 0.0024 \\
5 & $7.14 \%$ & 0.010 \\
29 & $41.43 \%$ & NS \\
21 & $30.00 \%$ & NS \\
\hline
\end{tabular}

$16.67 \%$

$24.07 \%$

$50.00 \%$

$26.92 \%$

$30.00 \%$

NS 


\begin{tabular}{|c|c|c|c|c|c|c|c|c|c|}
\hline \multirow[b]{2}{*}{ Characteristics } & \multicolumn{3}{|c|}{$\begin{array}{l}\text { Pre-Bundle NEC } \\
\mathrm{N}=54\end{array}$} & \multicolumn{5}{|c|}{$\begin{array}{l}\text { Post-Bundle NEC } \\
\qquad N=70\end{array}$} & \multirow[b]{2}{*}{ P-value } \\
\hline & \multicolumn{8}{|c|}{ Demographics } & \\
\hline$\overline{\text { Gestation age at Birth (All) }}$ & 28.61 & ( & 3.87 & ) & 29.16 & ( & 4.65 & ) & NS \\
\hline 23 to 27 weeks & 26 & ( & $48.15 \%$ & ) & 33 & ( & $47.14 \%$ & ) & \\
\hline 28 to 30 weeks & 14 & ( & $25.93 \%$ & ) & 14 & ( & $20.00 \%$ & ) & NS \\
\hline$\geq 31$ weeks & 14 & ( & $25.93 \%$ & ) & 23 & ( & $32.86 \%$ & ) & \\
\hline Birth Weight (All) & 1257.31 & ( & 686.61 & ) & 1391.14 & ( & 931.54 & ) & NS \\
\hline 23 to 27 weeks & 836.19 & ( & 214.24 & ) & 772.45 & ( & 168.64 & ) & NS \\
\hline 28 to 30 weeks & 1158.21 & ( & 261.81 & ) & 1044.71 & ( & 361.40 & ) & NS \\
\hline$\geq 31$ weeks & 2138.50 & ( & 750.79 & ) & 2489.70 & ( & 831.18 & ) & NS \\
\hline Male Gender & 32 & & $59.26 \%$ & & 41 & & $58.57 \%$ & & NS \\
\hline Days of life at NEC diagnosis & 16.44 & ( & 11.61 & ) & 25.10 & ( & 26.76 & ) & NS \\
\hline \multicolumn{10}{|l|}{ Prenatal factors } \\
\hline Cocaine/Methamphetamine use & 3 & & $5.56 \%$ & & 4 & & $6.56 \%$ & & NS \\
\hline Tobacco use & 9 & & $16.98 \%$ & & 4 & & $6.56 \%$ & & NS \\
\hline PIH/Preeclampsia/HTN & 8 & & $14.81 \%$ & & 14 & & $22.95 \%$ & & NS \\
\hline Chorioamnionitis & 6 & & $11.11 \%$ & & 3 & & $5.00 \%$ & & NS \\
\hline Indomethacin & 4 & & $7.41 \%$ & & 4 & & $6.45 \%$ & & NS \\
\hline Betamethasone & 36 & & $66.67 \%$ & & 39 & & $60.94 \%$ & & NS \\
\hline Fetal Distress & 9 & & $16.67 \%$ & & 11 & & $18.33 \%$ & & NS \\
\hline Multiple gestation & 16 & & $29.63 \%$ & & 16 & & $23.88 \%$ & & NS \\
\hline \multicolumn{10}{|l|}{ Co-morbidities } \\
\hline$\overline{\text { Pneumothorax }}$ & 4 & & $7.41 \%$ & & 2 & & $3.03 \%$ & & NS \\
\hline PDA & 21 & & $38.89 \%$ & & 34 & & $50.75 \%$ & & NS \\
\hline PDA Ligation & 10 & & $18.52 \%$ & & 13 & & $19.40 \%$ & & NS \\
\hline GI anomal & 2 & & $3.70 \%$ & & 12 & & $17.91 \%$ & & 0.020 \\
\hline IVH & 21 & & $38.89 \%$ & & 21 & & $31.82 \%$ & & NS \\
\hline IUGR/SGA & 3 & & $5.26 \%$ & & 11 & & $16.92 \%$ & & NS \\
\hline CV Abnormalities & 4 & & $7.41 \%$ & & 4 & & $6.25 \%$ & & NS \\
\hline \multicolumn{10}{|l|}{ NEC Care Bundle interventions } \\
\hline Received trophic feeds & 32 & & $60.38 \%$ & & 48 & & $72.73 \%$ & & NS \\
\hline Days of trophic feeds & 3.38 & ( & 2.42 & ) & 4.59 & ( & 2.73 & ) & NS \\
\hline \multicolumn{10}{|l|}{ Types of trophic Feed } \\
\hline MBM only & 8 & ( & $25.00 \%$ & ) & 39 & ( & $84.78 \%$ & ) & \\
\hline MBM/DBM & 0 & ( & $0.00 \%$ & ) & 5 & ( & $10.87 \%$ & ) & \\
\hline MBM/Formula & 11 & ( & $34.38 \%$ & ) & 0 & ( & $0.00 \%$ & ) & $<0.0001$ \\
\hline MBM/DBM/Formula & 0 & ( & $0.00 \%$ & ) & 1 & ( & $2.17 \%$ & ) & \\
\hline Formula only & 13 & ( & $40.63 \%$ & 1 & 1 & ( & $2.17 \%$ & ) & \\
\hline Non-trophic Feeds & 46 & & $85.19 \%$ & & 54 & & $83.08 \%$ & & NS \\
\hline \multicolumn{10}{|l|}{ Feeds Type } \\
\hline MBM only & 3 & ( & $6.52 \%$ & ) & 27 & ( & $51.92 \%$ & ) & \\
\hline MBM/DBM & 0 & ( & $0.00 \%$ & ) & 3 & ( & $5.77 \%$ & ) & \\
\hline MBM/Formula & 31 & ( & $67.39 \%$ & ) & 15 & ( & $28.85 \%$ & ) & $<0.0001$ \\
\hline MBM/DBM/Formula & 0 & ( & $0.00 \%$ & ) & 2 & i & $3.85 \%$ & ) & \\
\hline Formula only & 12 & ( & $26.09 \%$ & ) & 5 & ( & $9.62 \%$ & ) & \\
\hline PRBC & 29 & & $53.70 \%$ & & 36 & & $51.43 \%$ & & NS \\
\hline Antibiotic use prior to NEC & 38 & & $70.37 \%$ & & 43 & & $62.32 \%$ & & NS \\
\hline Days antibiotics, 1st week of life & 3.20 & ( & 2.27 & ) & 3.23 & ( & 2.63 & ) & NS \\
\hline Indocin & 22 & & $40.74 \%$ & & 1 & & $1.56 \%$ & & $<0.0001$ \\
\hline \multicolumn{10}{|l|}{ Other Interventions } \\
\hline Hydrocortisone & 5 & & $9.26 \%$ & & 7 & & $11.29 \%$ & & NS \\
\hline Dexamethasone & 2 & & $3.70 \%$ & & 6 & & $9.23 \%$ & & NS \\
\hline Epogen & 14 & & $25.93 \%$ & & 4 & & $6.15 \%$ & & 0.0040 \\
\hline Ferrous sulfate & 16 & & $30.77 \%$ & & 16 & & $24.24 \%$ & & NS \\
\hline Ranitidine & 6 & & $11.11 \%$ & & 4 & & $6.45 \%$ & & NS \\
\hline Caffeine & 23 & & $42.59 \%$ & & 37 & & $56.92 \%$ & & NS \\
\hline Dopamine & 18 & & $33.33 \%$ & & 18 & & $27.69 \%$ & & NS \\
\hline Dobutamine & 1 & & $1.85 \%$ & & 3 & & $4.76 \%$ & & NS \\
\hline Epinephrine & 1 & & $1.85 \%$ & & 4 & & $6.35 \%$ & & NS \\
\hline
\end{tabular}

Abbreviations: NEC, necrotizing enterocolitis, NS, non-significant, PIH, pregnancy induced hypertension, HTN, hypertension, PDA, patent ductus arteriosus, GI, gastrointestinal, IVH, intraventricular hemorrhage, IUGR, intrauterine growth restriction, SGA, small for gestation, CV, cardiovascular, MBM, maternal breast milk, DBM, donor breast milk, PRBC, packed red blood cells. 


\begin{tabular}{|c|c|c|c|c|c|c|c|c|c|}
\hline \multicolumn{9}{|c|}{ Table 3 Comparison of Pre-Bundle NEC and Pre-Bundle Control groups } & \multirow{4}{*}{$\begin{array}{l}\text { P-value } \\
\text { (paired) }\end{array}$} \\
\hline & \multirow{2}{*}{\multicolumn{4}{|c|}{$\begin{array}{l}\text { Pre-Bundle NEC } \\
\quad N=54\end{array}$}} & \multirow{2}{*}{\multicolumn{4}{|c|}{$\begin{array}{l}\text { Pre-Bundle Control } \\
\qquad N=105\end{array}$}} & \\
\hline Total & & & & & & & & & \\
\hline Characteristics & \multicolumn{2}{|l|}{ Mean/Freq. ( } & $S D / \%$ & \multirow[t]{2}{*}{1} & \multirow[t]{2}{*}{ Mean/Freq. } & \multirow{2}{*}{\multicolumn{2}{|c|}{$S D / \%$}} & \multirow[t]{2}{*}{1} & \\
\hline Demographics & & & & & & & & & \\
\hline Gestation age at Birth (All) & 28.61 & ( & 3.87 & ) & 28.77 & ( & 4.03 & ) & NS \\
\hline 23 to 27 weeks & 26 & ( & $48.15 \%$ & ) & 48 & & $45.71 \%$ & ) & \\
\hline 28 to 30 weeks & 14 & ( & $25.93 \%$ & ) & 28 & ( & $26.67 \%$ & ) & NS \\
\hline$\geq 31$ weeks & 14 & ( & $25.93 \%$ & ) & 29 & ( & $27.62 \%$ & ) & \\
\hline Male Gender & 32 & ( & $59.26 \%$ & ) & 63 & ( & $60.00 \%$ & ) & NS \\
\hline \multicolumn{10}{|l|}{ Prenatal factors } \\
\hline Cocaine/Methamphtamine use & 3 & ( & $5.56 \%$ & ) & 6 & ( & $5.71 \%$ & ) & NS \\
\hline Tobacco use & 9 & ( & $16.98 \%$ & ) & 12 & ( & $11.43 \%$ & ) & NS \\
\hline PIH/Preeclampsia/HTN & 8 & ( & $14.81 \%$ & ) & 14 & ( & $13.33 \%$ & ) & NS \\
\hline Chorioamnionitis & 6 & ( & $11.11 \%$ & ) & 5 & ( & $4.76 \%$ & ) & NS \\
\hline Indomethacin & 4 & ( & $7.41 \%$ & ) & 6 & ( & $5.71 \%$ & ) & NS \\
\hline Betamethasone & 36 & ( & $66.67 \%$ & ) & 67 & ( & $63.81 \%$ & ) & NS \\
\hline Fetal Distress & 9 & ( & $16.67 \%$ & 1 & 23 & ( & $21.90 \%$ & ) & NS \\
\hline Multiple gestation & 16 & ( & $29.63 \%$ & ) & 24 & ( & $22.86 \%$ & ) & NS \\
\hline \multicolumn{10}{|l|}{ Co-morbidities } \\
\hline Pneumothorax & 4 & ( & $7.41 \%$ & ) & 2 & ( & $1.90 \%$ & ) & NS \\
\hline PDA & 21 & ( & $38.89 \%$ & ) & 39 & ( & $37.14 \%$ & ) & NS \\
\hline PDA Ligation & 10 & ( & $18.52 \%$ & ) & 12 & ( & $11.43 \%$ & ) & NS \\
\hline Gl anomal & 2 & ( & $3.70 \%$ & ) & 3 & ( & $2.86 \%$ & ) & NS \\
\hline IVH & 21 & ( & $38.89 \%$ & ) & 24 & ( & $22.86 \%$ & ) & 0.034 \\
\hline IUGR/SGA & 3 & ( & $5.56 \%$ & ) & 4 & ( & $3.81 \%$ & ) & NS \\
\hline CV Abnor & 4 & ( & $7.41 \%$ & ) & 2 & ( & $1.90 \%$ & ) & NS \\
\hline \multicolumn{10}{|l|}{ NEC Care Bundle interventions } \\
\hline Received trophic feeds & 32 & ( & $60.38 \%$ & ) & 58 & ( & $55.24 \%$ & ) & NS \\
\hline Days of trophic feeds & 3.38 & ( & 2.42 & ) & 3.66 & ( & 3.14 & ) & NS \\
\hline \multicolumn{10}{|l|}{ Types of trophic Feed } \\
\hline MBM only & 8 & ( & $25.00 \%$ & ) & 25 & ( & $43.10 \%$ & ) & \\
\hline MBM/DBM & 0 & ( & $0.00 \%$ & ) & 0 & ( & $0.00 \%$ & ) & \\
\hline MBM/Formula & 11 & ( & $34.38 \%$ & ) & 18 & ( & $31.03 \%$ & ) & NS \\
\hline MBM/DBM/Formula & 0 & ( & $0.00 \%$ & ) & 0 & ( & $0.00 \%$ & ) & \\
\hline Formula only & 13 & ( & $40.63 \%$ & ) & 15 & ( & $25.86 \%$ & ) & \\
\hline Non-trophic Feeds & 46 & ( & $85.19 \%$ & ) & 94 & ( & $89.52 \%$ & ) & NS \\
\hline \multicolumn{10}{|l|}{ Feeds Type } \\
\hline MBM only & 3 & ( & $6.52 \%$ & ) & 11 & 1 & $11.70 \%$ & ) & \\
\hline MBM/DBM & 0 & ( & $0.00 \%$ & ) & 0 & ( & $0.00 \%$ & ) & \\
\hline MBM/Formula & 31 & ( & $67.39 \%$ & ) & 66 & ( & $70.21 \%$ & ) & NS \\
\hline MBM/DBM/Formula & 0 & 1 & $0.00 \%$ & ) & 0 & ( & $0.00 \%$ & ) & \\
\hline Formula only & 12 & i & $26.09 \%$ & ) & 17 & ( & $18.09 \%$ & ) & \\
\hline PRBC & 25 & 1 & $46.30 \%$ & ) & 50 & ( & $47.62 \%$ & ) & NS \\
\hline Antibiotics & 38 & ( & $70.37 \%$ & ) & 72 & ( & $68.57 \%$ & ) & NS \\
\hline Days antibiotics, 1st week of life & 3.20 & ( & 2.27 & ) & 3.41 & ( & 3.06 & ) & NS \\
\hline Indocin & 22 & ( & $40.74 \%$ & ) & 34 & ( & $32.38 \%$ & ) & NS \\
\hline
\end{tabular}

Abbreviations: NEC, necrotizing enterocolitis, NS, non-significant, PIH, pregnancy induced hypertension, HTN, hypertension, PDA, patent ductus arteriosus, GI, gastrointestinal, IVH, intraventricular hemorrhage, IUGR, intrauterine growth restriction, SGA, small for gestation, CV, cardiovascular, MBM, maternal breast milk, DBM, donor breast milk, TPN, total parenteral nutrition, PRBC, packed red blood cells. 
Table 4 Comparison of Post-Bundle NEC and Post-Bundle Control groups

\section{Post-Bundle NEC}

Total

Characteristics

Demographics

Gestation age at Birth (All)

23 to 27 weeks

28 to 30 weeks

$\geq 31$ weeks

Male Gender

Prenatal factors

Cocaine/Methamphtamine use

Tobacco use

$\mathrm{PIH} /$ Preeclampsia/HTN

Chorioamnionitis

Indomethacin

Betamethasone

Fetal Distress

Multiple gestation

Co-morbidities

Pneumothorax

PDA

PDA Ligation

GI anomal

IVH

IUGR/SGA

CV Abnor

NEC Care Bundle interventions

Received trophic feeds
Days of trophic feeds
Types of trophic Feed
MBM only
MBM/DBM
MBM/Formula
MBM/DBM/Formula
Formula only
Non-trophic Feeds
Feeds Type
MBM only
MBM/DBM
MBM/Formula
MBM/DBM/Formula
Formula only
PRBC

$\mathrm{N}=70$

Post-Bundle Control

$\mathrm{N}=131$
P-value

Mean/Freq. (SD/\% ) Mean/Freq. (SD/\% ) (paired)

$\begin{array}{cccccc}29.16 & (4.65) & 29.41 & (4.70) \\ 33 & (47.14 \%) & 62 & (47.33 \% & ) \\ 14 & (20.00 \%) & 24 & (18.32 \% & ) \\ 23 & (32.86 \%) & 45 & (34.35 \%) \\ 41 & (58.57 \%) & 76 & (58.02 \%)\end{array}$

NS

NS

NS

$\begin{array}{ccccccc}4 & (6.56 \%) & 5 & (4.59 \%) & \text { NS } \\ 4 & (6.56 \%) & 4 & (3.74 \%) & \text { NS } \\ 14 & (22.95 \%) & 32 & (29.09 \%) & \text { NS } \\ 3 & (5.00 \%) & 11 & (10.09 \%) & \text { NS } \\ 4 & (6.45 \%) & 5 & (4.63 \%) & \text { NS } \\ 39 & (60.94 \%) & 70 & (63.06 \%) & \text { NS } \\ 11 & (18.33 \%) & 23 & (21.30 \%) & \text { NS } \\ 16 & (23.88 \%) & 17 & (15.32 \%) & \text { NS }\end{array}$

$\begin{array}{cccccc}2 & (3.03 \%) & 4 & (3.48 \%) & \text { NS } \\ 34 & (50.75 \%) & 37 & (30.83 \%) & 0.020 \\ 13 & (19.40 \%) & 12 & (10.00 \%) & \text { NS } \\ 12 & (17.91 \%) & 18 & (15.65 \%) & \text { NS } \\ 21 & (31.82) & 22 & (18.64 \%) & \text { NS } \\ 11 & (16.92 \%) & 13 & (11.50 \%) & \text { NS } \\ 4 & (6.25 \%) & 11 & (9.82 \%) & \text { NS }\end{array}$

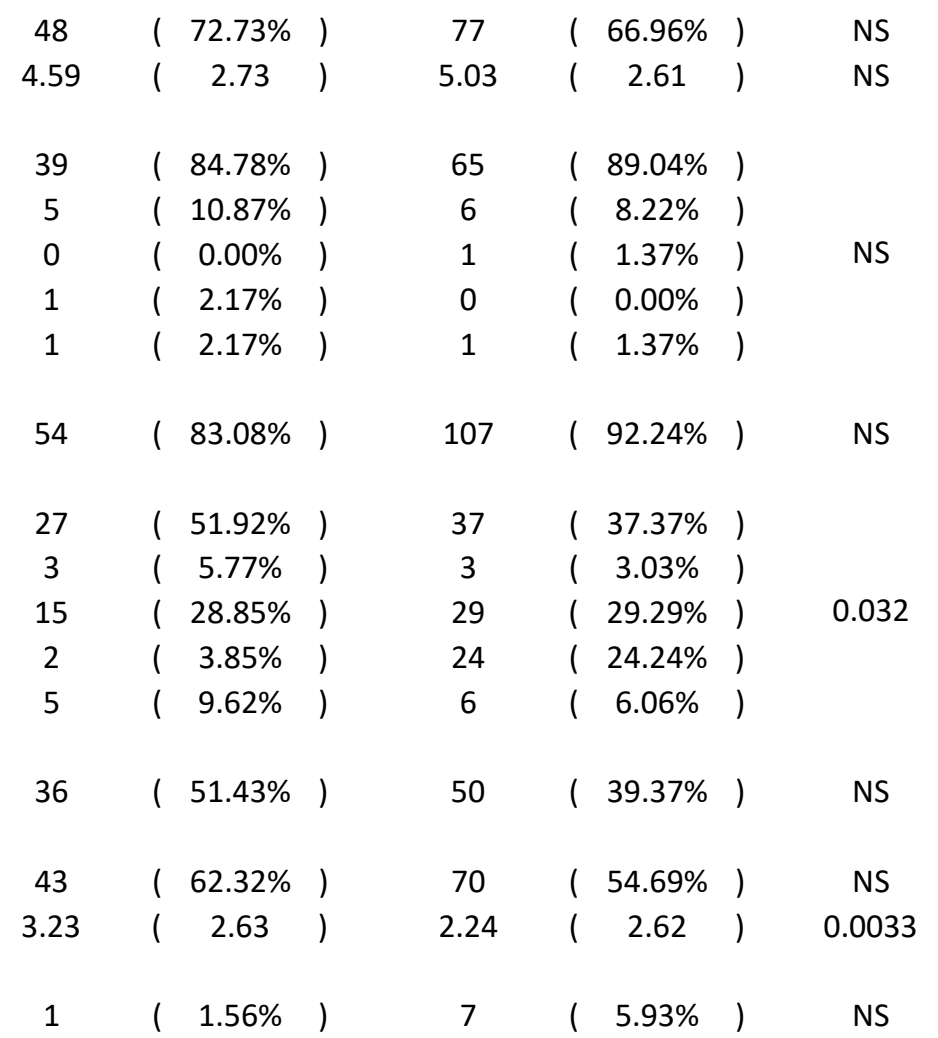

Abbreviations: NEC, necrotizing enterocolitis, NS, non-significant, $\mathrm{PIH}$, pregnancy induced hypertension, HTN, hypertension, PDA, patent ductus arteriosus, GI, gastrointestinal, IVH, intraventricular hemorrhage, IUGR, intrauterine growth restriction, SGA, small for gestation, CV, cardiovascular, MBM, maternal breast milk, DBM, donor breast milk, TPN, total parenteral nutrition, PRBC, packed red blood cells. 
Table 5 Comparison of Pre-Bundle Control and Post-Bundle Control groups

\section{Pre-Bundle Control}

$\mathrm{N}=105$
Post-Bundle Control

$\mathrm{N}=131$

$\begin{array}{cccccc}\text { Mean/Freq. } & (S D / \%) & \text { Mean/Freq. } & (S D / \%) \\ 28.77 & (4.03) & 29.41 & (4.70) \\ 48 & (45.71 \%) & 62 & (47.33 \%) \\ 28 & (26.67 \%) & 24 & (18.32 \%) \\ 29 & (27.62 \%) & 45 & (34.35 \%) \\ 1346.81 & (741.52) & 1471.27 & (926.75) \\ 808.49 & (208.83) & 833.48 & (173.86) \\ 1264.79 & (284.44) & 1174.46 & (242.40) \\ 2298.45 & (664.76) & 2508.29 & (862.47) \\ 63 & (60.00 \%) & 76 & (58.02 \%)\end{array}$

P-value

NS

NS

NS

NS

NS

NS

NS

NS

0.0015

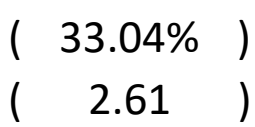

3.66
25

0

18

0

15

$\left.\begin{array}{l}\left(\begin{array}{c}43.10 \% \\ ( \\ 0.00 \%\end{array}\right) \\ (31.03 \% \\ \left(\begin{array}{c}0.00 \% \\ (\end{array}\right) \\ 25.86 \%\end{array}\right)$

94

11

MBM only

MBM/DBM

MBM/Formula

MBM/DBM/Formula

Formula only

PRBC

Antibiotics

Days antibiotics, 1st week of life
0

66

0

17

50

72

3.41

34
( $47.62 \%)$

( $11.70 \%)$

( $0.00 \%$ )

( $70.21 \%)$

( $0.00 \%$ )

( $18.09 \%$ )

$47.62 \%$

( 68.57\% )

$3.06)$

$32.38 \% \quad)$
38

65

6

1

0

1

107

37

3

29

24

6

50

70

2.24

7

Indocin

( $89.04 \%)$

( $8.22 \%$

( $1.37 \%$

( $0.00 \%)$

( $1.37 \%$ )

$<0.0001$

NS

$<0.0001$

NS

0.032

0.0009

Abbreviations: NEC, necrotizing enterocolitis, NS, non-significant, MBM, maternal breast milk, DBM, donor breast milk, PRBC, packed red blood cells. 
23-27 weeks gestation, $28-30$ weeks gestation, and $\geq 31$ weeks gestation.

Infants were initially identified by a diagnosis of NEC in the problem list or discharge summary in the electronic medical record. From this initial search, we then limited the group to infants that completed at least 7 days NPO with antibiotic treatment. Infants diagnosed with spontaneous intestinal perforation were excluded. We also excluded patients that were transferred to our NICU with a preexisting diagnosis of NEC. Controls for each case were selected, matched with birth year, gestational age, and gender. The demographic and clinical data were summarized using descriptive statistics.

Institutional review board approval was obtained for access to the electronic medical records. Multiple clinical and demographic data were evaluated. Fischer's exact test, chi-square test, KruskalWallis test, paired Wilcoxon signed-rank test, and exact conditional logistical regression were used for statistical analysis.

\section{Results}

During the study period, 124 patients met the criteria for NEC diagnosis, and their charts were reviewed. There were 54 patients in the Pre-Bundle cohort and 70 patients in the Post-Bundle cohort. In addition, 236 patient charts were reviewed as NEC controls, 105 in the Pre-Bundle group and 131 in the Post-Bundle group. Each cohort was further classified into three age groups as assigned at birth: 23-27 weeks gestation, 28-30 weeks gestation, and $\geq 31$ weeks gestation.

\section{Pre-Bundle NEC vs. Post-Bundle NEC}

The incidence of NEC in the Pre-Bundle and Post-Bundle cohorts is displayed in Table 1 . There was a $56.8 \%$ reduction in overall incidence of NEC in the Post-Bundle group compared to the Pre-Bundle group in our NICU. NEC incidence was $1.92 \%$ in the Pre-Bundle group and $0.83 \%$ in the Post-Bundle group ( $p$-value $<0.0001)$. This reduction in NEC was primarily seen in the $23-27$ weeks and 28-30 weeks gestation groups. In the 23-27 weeks gestation group, there was a $57.1 \%$ reduction in the incidence (14.21 to $6.09 \%$ ). The $28-30$ weeks gestation group had a $62.2 \%$ reduction (5.56 to $2.10 \%$ ). In the $\geq 31$ weeks gestation age group, there was a trend in NEC reduction that was not statistically significant. There was a significant reduction (16.67 to $1.43 \%$ ) of recurrent NEC in the Post-Bundle NEC group. There was a significant reduction in transfusion-associated NEC in the Post-Bundle group from 24.07 to $7.14 \%$. We define transfusion-associated NEC as NEC occurring within 72 hours after initiation of a PRBC transfusion. There was no significant difference in the incidence of surgical NEC or deaths associated with NEC between the two historical cohorts.
Table 2 compares demographic, maternal, and infant characteristics between Pre-Bundle and Post-Bundle NEC groups. There were no significant differences in gestational age at birth, birth weight, gender, or maternal conditions. In the Post-Bundle group, there was increased use of MBM and DBM and reduced preterm infant formula for both trophic and non-trophic feedings. Interestingly, the Post-Bundle NEC group had a higher incidence of gastrointestinal anomalies (17.91 vs. $3.70 \%$ ) compared to the PreBundle NEC group. There were no significant differences in the incidence of IVH, intrauterine growth restriction (IUGR), small for gestational age (SGA), or other congenital or cardiac anomalies. In the Post-Bundle NEC group, there was a significant reduction in the use of indomethacin and erythropoietin.

\section{NEC vs Control}

Demographic, maternal, and infant characteristics comparing each NEC cohort with its respective control group are listed in Tables 3 and 4 . There were no significant differences in gestational age at birth, gender, maternal conditions, or perinatal factors between the NEC and control groups in either the Pre-Bundle or Post-Bundle cohorts. There were no significant differences in the use of MBM, DBM, PRBC transfusions, or indomethacin use in either the Pre-Bundle or Post-Bundle cohorts.

There was a higher incidence of IVH $(P=0.034)$ in the Pre-Bundle NEC group compared to Pre-Bundle Control. There was a higher incidence of PDA $(P=0.020)$ in the Post-Bundle NEC group compared to Post-Bundle Control. There was also a significantly higher mean days of antibiotics during the first week of life in the Post-Bundle NEC group compared to the Post-Bundle Control ( $P$ $=0.003$ ).

\section{Pre-Bundle Control vs. Post-Bundle Control}

The changes in practice before and after implementing the NEC care bundle are displayed in Table 5, which compares the PreBundle and Post-Bundle control groups. There was no difference between the two groups with the implementation of trophic feeds. However, the duration of trophic feedings increased from mean of 3.66 to 5.03 days $(P=0.0015)$. There was also a significant change in practice for the type of feeding given to patients, with increased use of MBM and DBM and reduction in the use of preterm infant formula. In the Post-Bundle control group, there was a significant reduction in the use of antibiotics 68.57 to $54.69 \%(P=$ 0.032 ) as well as a reduction in the mean number of days of early empiric antibiotics in culture-negative infants, 3.06 to 2.24 days $(P=0.0009)$.

There was a significant decrease in indomethacin from 32.38 to $5.93 \%(P<0.0001)$. We did not see a change in PRBC transfusions between the two control groups.

\section{"After implementing an NEC care bundle, there was a significant reduction in the incidence of NEC from 14.21 to $6.09 \%$ in our 23-27 weeks gestation infants and a reduction from 5.56 to $2.10 \%$ in our $28-30$ weeks gestation infants."}

\section{Discussion}

After implementing a NEC care bundle, there was a significant reduction in the incidence of NEC from 14.21 to $6.09 \%$ in our $23-$ 27 weeks gestation infants and a reduction from 5.56 to $2.10 \%$ in 
our 28-30 weeks gestation infants. In the ten-year Post-Bundle cohort, we had one patient with recurrent NEC, an incidence of $1.43 \%$, compared to a recurrent NEC incidence of $16.67 \%$ in the Pre-Bundle group. We also saw a reduction in transfusion-associated NEC from $24.07 \%$ in the Pre-Bundle group to $7.14 \%$ in the Post-Bundle group.

When the NEC care bundle was implemented in our NICU, it was done in a stepwise approach over a 12 month period. Each intervention, when combined, had a cumulative effect that would not have been achieved were each component implemented alone. Although the use of protocols that decrease the incidence of NEC has been described previously, this is the first study, to our knowledge, that evaluates the impact of a NEC care bundle on both incidence and recurrence of NEC.

We suggest that several factors played a significant role in decreasing NEC incidence in our NICU. Implementation of a standardized feeding protocol eliminated variation in feeding practices within our group. In addition, using an exclusive human milk policy supported by the adoption of a donor human milk program virtually eliminated the use of preterm formula in our VLBW population. Implementation of a standardized transfusion protocol eliminated variation in transfusion practices, minimized unnecessary transfusions, and exposure to high-risk transfusions with severe anemia. Limiting empiric antibiotic therapy duration also impacted the unnecessary use of antibiotics in infants with low-risk factors and few clinical signs of sepsis at admission. Lastly, stopping the use of indomethacin in the NICU eliminated the associated risks for feeding and gastrointestinal complications without increasing PDA incidence of complications.

Previous studies support the importance of a standardized feeding protocol. Patole et al. concluded that the single most effective strategy to prevent NEC was implementing a standardized feeding regimen.(9) Standardized feeding regimens are beneficial since they decrease practice variations and increase awareness of potential feeding problems.(9) Our feeding protocol resulted in less variation in feeding advancements and fortification practices. Standardized feeding protocols have been found to be safe, resulting in the earlier achievement of full enteral feeds, reduced time on hyperalimentation therapy, better growth outcomes, and decreased length of stay.(25-27)

\section{"Implementation of early empiric antibiotic treatment in preterm infants born for non-maternal reasons is a common practice because of this population's immature immune system and risk for morbidity and mortality from early-onset sepsis. Duration of treatment is often based on individual practice and NICU policy as opposed to strong evidence-based practices."}

Our donor milk program was started to support infants whose mothers did not have enough MBM. It has been well documented that feeding preterm infants an exclusive human milk diet decreases NEC. $(15,16,28-31)$ When there is insufficient MBM available, $\mathrm{DBM}$ is the preferred alternative for preventing NEC. A Cochrane meta-analysis review comparing formula milk versus donor milk for feeding preterm infants showed a higher risk of developing NEC with formula-fed infants.(32)

Although controversial, several studies have described an association between PRBC transfusion and NEC.(2,33-35) The etiology of transfusion-associated NEC or gut injury remains unclear. The association may be related to the level of anemia,(2) inflammatory factors associated with transfusion, or a combination of both. It has been suggested that withholding feeds during the transfusion may be protective.(34) As an alternative to completely withholding enteral feedings during transfusions, we chose to provide trophic feedings during this time period. In large part, we adopted a transfusion protocol due to observations and experience with transfusion-associated NEC in our NICU. This decision was reinforced by the significant reduction in the number of transfusion-associated gastrointestinal and NEC events seen in our Post-Bundle NEC cohort.

Implementation of early empiric antibiotic treatment in preterm infants born for non-maternal reasons is a common practice because of this population's immature immune system and risk for morbidity and mortality from early-onset sepsis. Duration of treatment is often based on individual practice and NICU policy as opposed to strong evidence-based practices. It is generally accepted that empiric antibiotics are safe, with perceived benefits outweighing potential risks. This is evident in the common practice of treating culture-negative sepsis.(36) However, emerging studies have reported that prolonged initial empiric antibiotic treatment ( $>5$ days) may be associated with adverse outcomes, including late-onset sepsis and death, and that each additional day of antibiotic use increases the risk of NEC.(37-39) Because of the increased morbidity associated with prolonged empiric antibiotic treatment, we reduced our use of early empiric treatment from 72 to 48 hours for infants with sterile culture results. To date, the use of any empiric antibiotics in our preterm population has decreased significantly as a result of these awareness practices.

In the NICU, indomethacin has been used both as prophylaxis for $\mathrm{IVH}$ and PDA and as a medical treatment for PDA closure. Studies report an increased risk of spontaneous perforation and NEC with prophylactic and therapeutic indomethacin use in preterm infants. $(40,41)$ In a large retrospective study, O'Donovan et al. reported that indomethacin was not associated with an increased risk for NEC,(42) and although supported in a subsequent study, an increased risk for intestinal perforation with indomethacin was noted.(43) Prior to our NEC bundle, there was inconsistent practice using prophylactic indomethacin for IVH and PDA prophylaxis. With the NEC bundle implementation, routine use of prophylactic indomethacin was discontinued, and ibuprofen became the drug of choice for medical treatment of a symptomatic PDA. We did not see a significant change in PDA incidence or number of PDA ligations in the Pre-Bundle Control and Post-Bundle Control groups.

Our NEC care bundle was instrumental in decreasing the incidence and recurrence of NEC in a NICU with an already relatively low NEC rate. Balancing protocol-driven care for routine and common decisions with the autonomy for individualized care is important for physician buy-in and adherence to the care bundle. The strength of adherence to the NEC care bundle in our NICU is supported by the significant changes seen in the Pre-Bundle and Post-Bundle control group comparison results.

Limitations of our study should be noted. First and most importantly, we describe the implementation of a bundle specific to our NICU population. Generalization of this bundle to other NICU populations may not necessarily generate similar results. Secondly, our organization transitioned EMR systems between our Pre-Bundle and Post-Bundle study periods. As a result, there were missing or unavailable data for some analysis in the early Post-Bundle chart reviews. Finally, the protocols were introduced 
stepwise to improve compliance, so the early Post-Bundle group did not reflect the full impact of all the protocols. We also did not measure the impact of each protocol individually. Future studies could evaluate the impact of each protocol separately.

\section{"This decreased incidence of NEC was attained after implementing an NEC care bundle that centered on a standardized feeding protocol that relied primarily on a human milk-based diet, along with protocols for PRBC transfusions, the use of empiric antibiotics, and indomethacin."}

\section{Conclusion}

Implementation of a NEC care bundle decreased NEC incidence in our NICU population, with the greatest impact seen in the most vulnerable preterm and very preterm infants. Incidence of recurrent NEC and transfusion-associated NEC were significantly decreased. This decreased incidence of NEC was attained after implementing a NEC care bundle that centered on a standardized feeding protocol that relied primarily on a human milk-based diet, along with protocols for PRBC transfusions, the use of empiric antibiotics, and indomethacin.

\section{References:}

1. Singh R, Visintainer P, Frantz III I, Shah BL, Meyer KM, Favila $S A$ et al. Association of necrotizing enterocolitis with anemia and packed red blood cell transfusions of preterm infants. Journal of Perinatology 2011; 31(3): 176-182.

2. Battersby C, Santhalingam T, Costeloe K, Modi N. Incidence of neonatal necrotising enterocolitis in high-income countries: a systematic review. Arch Dis Child Fetal Neonatal Ed. 2018; 103(2):F182-F189.

3. Stringer MD, Brereton RF, Drake DP, Kiely EM, Capps SN, Spitz L. Recurrent necrotizing enterocolitis. Journal of Pediatric Surgery 1993; 28(8): 979-981.

4. Thyoka M, Eaton S, Hall NJ, Drake D, Kiely E, Curry J et al. Advanced necrotizing enterocolitis part 2: recurrence of necrotizing enterocolitis. European Journal of Pediatric Surgery 2012; 22(1): 13-16.

5. Ehrenkranz RA, Dusick AM, Vohr BR, Wright LL, Wrage LA, Poole WK. Growth in the neonatal intensive care unit influences neurodevelopmental and growth outcomes of extremely low birth weight infants. Pediatrics 2006; 117: 12531261.

6. Lucas A, Morley R, Cole TJ. Randomised trial of early diet in preterm babies and later intelligence quotient. BMJ 1998; 317: 1481-1487.

7. Chan SHT, Johnson MJ, Leaf AA, Vollmer B. Nutrition and neurodevelopmental outcomes in preterm infants: a systematic review. Acta Paediatrica 2016; 105: 587-599.

8. Young L, Morgan J, McGuire W. Preventing necrotizing enterocolitis in very low birth weight infants: current evidence. Paediatrics and Child Health 2010; 21(6): 258-264.

9. Patole SK, de Klerk N. Impact of standardized feeding regimens on incidence of neonatal necrotising enterocolitis: a systematic review and meta-analysis of observational studies. Arch Dis Child Fetal Neonatal Ed 2005; 90: F147-F151.

10. Gephart SM, Hanson CK. Preventing necrotizing enterocolitis with standardized feeding protocols. Advances in Neonatal Care 2013; 13(1): 48-54.

11. Kamitsuka MD, Horton MK, Williams MA. The incidence of necrotizing enterocolitis after introducing standardized feeding schedules for infants between 1250 and 2500 grams and less than 35 weeks of gestation. Pediatrics 2000; 105: 379384.

12. Berseth CL, Bisquera JA, Paje VU. Prolonging small feeding volumes early in life decreases the incidence of necrotizing enterocolitis in very low birth weight infants. Pediatrics 2003; 111: 529-534.

13. Colaizy TT, Bartick MC, Jegier BJ, Green BD, Reinhold AG, Schaefer AJ et al. Impact of optimized breastfeeding on the costs of necrotizing enterocolitis in extremely low birthweight infants. The Journal of Pediatrics 2016; 175: 100-105.

14. Sisk PM, Lovelady CA, Dillard RG, Gruber KJ, O'shea TM. Early human milk feeding is associated with a lower risk of necrotizing enterocolitis in very low birth weight infants. Journal of Perinatology 2007; 27(7): 428-433.

15. Herrmann K, Carroll K. An exclusively human milk diet reduces necrotizing enterocolitis. Breastfeeding medicine 2014; 9: 184-190.

16. Sullivan S, Schanler RJ, Kim JH, Patel AL, Trawöger R, Kiechl-Kohlendorfer $U$ et al. An exclusively human milkbased diet is associated with a lower rate of necrotizing enterocolitis than a diet of human milk and bovine milk-based products. The Journal of Pediatrics 2010; 156(4):562-567.

17. McGuire A, Anthony MY. Donor human milk versus formula for preventing necrotizing enterocolitis in preterm infants: systematic review. Arch Dis Child Fetal Neonatal Ed 2003; 88: 11-14.

18. Haraden C. What is a Bundle? IHI Web site Improvement Stories. http://www.ihi.org/resources/Pages/ImprovementStories/What/saBundle. aspx. Published April 26, 2011.

19. Resar $R$, Pronovost $P$, Haraden $C$. Using a bundle approach to improve ventilator care processes and reduce ventilatorassociated pneumonia. Jt Comm J Qual Patient Saf 2005; 31: 243-48.

20. Weber C. Applying adult ventilator-associated pneumonia bundle evidence to the ventilated neonate. Advances in Neonatal Care. 2016; 16(3): 178-190.

21. Pogorzelska-Maziarz M. The use and effectiveness of bundles for prevention of central line-associated bloodstream

NEONATOLOGY TODAY is interested in publishing manuscripts from Neonatologists, Fellows, NNPs and those involved in caring for neonates on case studies, research results, hospital news, meeting announcements, and other pertinent topics. Please submit your manuscript to: LomaLindaPublishingCompany@gmail.com 
infections in neonates. J Perinat Neonat Nurs 2016; 30(2): 148-159.

22. Steiner M, Langgartner M, Cardona F, Waldhör T, Schwindt $J$, Haiden $J$ et al. Significant reduction of catheter-associated blood stream infections in preterm neonates after implementation of a care bundle focusing on simulation training of central line insertion. The Pediatric Infectious Disease Journal 2015; 34(11); 1193-1196.

23. Melish M, Carey-Goo P, Delaney H, Neal C, Tao K, Kimata C et al. Reducing nosocomial infections in the nicu. American Journal of Infection Control 2013; S5-S6 (oral abstract 006).

24. Talavera MM, Bixler G, Cozzi C, Dail J, Miller RR, McClead Jr R et al. Quality improvement initiative to reduce the necrotizing enterocolitis rate in premature infants. Pediatrics 2016; 137(5): e1-e8.

25. McCallie KR, Lee HC, Mayer O, Cohen RS, Hintz SR, Rhine $W D$. Improved outcomes with a standardized feeding protocol for very low birth weight infants. Journal of Perinatology 2011; 31: S61-S67.

26. Butler TJ, Szekely LJ, Grow JL. A standardized nutrition approach for very low birth weight neonates improves outcomes, reduces cost and is not associated with increased rates of necrotizing enterocolitis, sepsis or mortality. Journal of Perinatology 2013; 33: 851-857.

27. Kuzma-O'Reilly B, Duenas ML, Greecher C, Kimberlin L, Mujsce D, Miller D et al. Evaluation, development, and implementation of potentially better practices in neonatal intensive care nutrition. Pediatrics 2014; 111(4): e461-e470.

28. Lucas A, Cole TJ. Breast milk and neonatal necrotizing enterocolitis. The Lancet 1990; 336(8730): 1519-1523.

29. Patel AL, Meier PP, Engstrom JL. The evidence for use of human milk in very low-birthweight preterm infants. NeoReviews 2007; 8(11): e459-e466.

30. Shulhan J, Dicken B, Hartling L, Larsen BMK. Current knowledge of necrotizing enterocolitis in preterm infants and the impact of different types of enteral nutrition products. Advances in Nutrition 2017; 8(1): 80-91.

31. Miller J, Tonkin E, Damarell RA, McPhee AJ, Suganuma $M$, Suganuma $H$, Middleton PF, Makrides M, Collins CT. A systematic review and meta-analysis of human milk feeding and morbidity in very low birth weight infants. Nutrients 2018; 10(6), 707

32. Quigley M, McGuire W. Formula milk versus donor breast milk for feeding preterm or low birth weight infants. Cochrane Database of Systematic Reviews 2014; 4: Art. No.:CD002971. DOI:10.1002/14651858.CD002971.pub3.

33. Mohamed A, Shah P. Transfusion associated necrotizing enterocolitis: a meta-analysis of observational data. Pediatrics 2012; 129: 529-540.

34. El-Dib M, Narang S, Lee E, Massaro AN, Aly H. Red blood cell transfusion, feeding and necrotizing enterocolitis in preterm infants. Journal of Perinatology 2011; 31: 183-187.

35. Paul DA, Mackley A, Novitsky A, Zhao Y, Brooks A, Locke $R G$. Increased odds of necrotizing enterocolitis after transfusion of red blood cells in premature infants. Pediatrics 2011; 127(4): 635-641.

36. Cantey JB, Baird SD. Ending the culture of culture-negative sepsis in the neonatal icu. Pediatrics 2017; 140(4): 1-4.

37. Cotton CM, Taylor S, Stoll B, Goldberg RN, Hansen NI, Sánchez $P J$ et al. Prolonged duration of initial empirical antibiotic treatment is associated with increased rates of necrotizing enterocolitis and death for extremely low birth weight infants. Pediatrics 2009; 123: 58-66.

38. Kuppala VS, Meinzen-Derr J, Morrow AL, Schibler KR. Prolonged initial empirical antibiotic treatment is associated with adverse outcomes in premature infants. J Pediatr 2011; 159(5): 720-725.

39. Alexander VN, Northrup V, Bizzarro J. Antibiotic exposure in the newborn intensive care unit and the risk of necrotizing enterocolitis. The Journal of Pediatrics 2011; 159(3): 392-397.

40. Grosfeld JL, Chaet M, Molinari F, Engle W, Engum SA, West $K W$ et al. Increased risk of necrotizing enterocolitis in premature infants with patent ductus arteriosus treated with indomethacin. Annals of Surgery 1996; 224(3): 350-357.

41. Fujii AM, Brown E, Mirochnick M, O'Brien S, Kaufman G. Neonatal necrotizing enterocolitis with intestinal perforation in extremely premature infants receiving early indomethacin treatment for patent ductus arteriosus. Journal of Perinatology 2002; 22: 535-540.

42. O'Donovan DJ, Baetiong A, Adams K, Chen A, O'Brian Smith $E$, Adams JM et al. Necrotizing enterocolitis and gastrointestinal complications after indomethacin therapy and surgical ligation in premature infants with patent ductus arteriosus. Journal of Perinatology 2003; 23: 286-290.

43. McPherson C, Gal P, Smith M, Wilder W, Richter S, Ransom JL et al. Necrotizing enterocolitis in preterm infants with patent ductus arteriosus: does indomethacin increase risk? Journal of Neonatal-Perinatal Medicine 2008; 1(4): 209-216.

Funding Sources: No funding has been provided to support this research.

Conflicts of Interest: The authors declare no conflicts of interest.

Author Contributions: All authors contributed to the writing and the review of this paper

NT
New subscribers are always welcome! NEONATOLOGY
To sign up for a free monthly subscription, just click on this box to go directly to our subscription page 


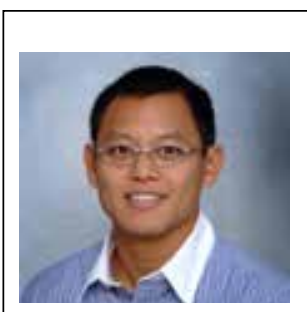

\section{Corresponding Author}

Jerome P. Lee, MD

Neonatal Hospitalist,

Hawai'i Pacific Health

Neonatal Intensive Care Unit

1319 Punahou Street

Honolulu, HI 96826

Email:jeromel@hphmg.org

808-983-6392 (fax)

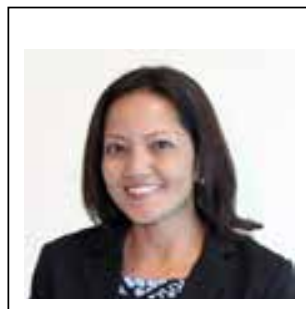

Winona K. Lee, MD

University of Hawai'i

John A. Burns School of Medicine

Mānoa 651 Illalo Street

Honolulu, Hawai'i 96813

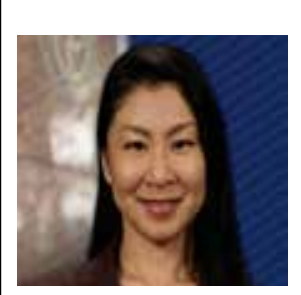

Chieko Kimata, PhD, MPH, MBA

Hawai'i Pacific Health

Neonatal Intensive Care Unit

1319 Punahou Street

Honolulu, HI 96826

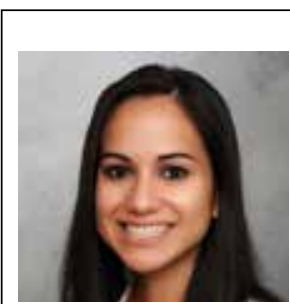

Alyssa Honda, MD

University of Hawai'i

John A. Burns School of Medicine

Mānoa 651 Illalo Street

Honolulu, Hawai'i 96813

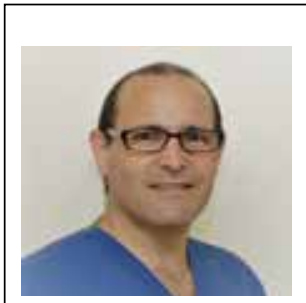

Charles R. Macías Neal, MD, PhD

University of Hawai'i

John A. Burns School of Medicine

Mānoa 651 Illalo Street

Honolulu, Hawai'i 96813

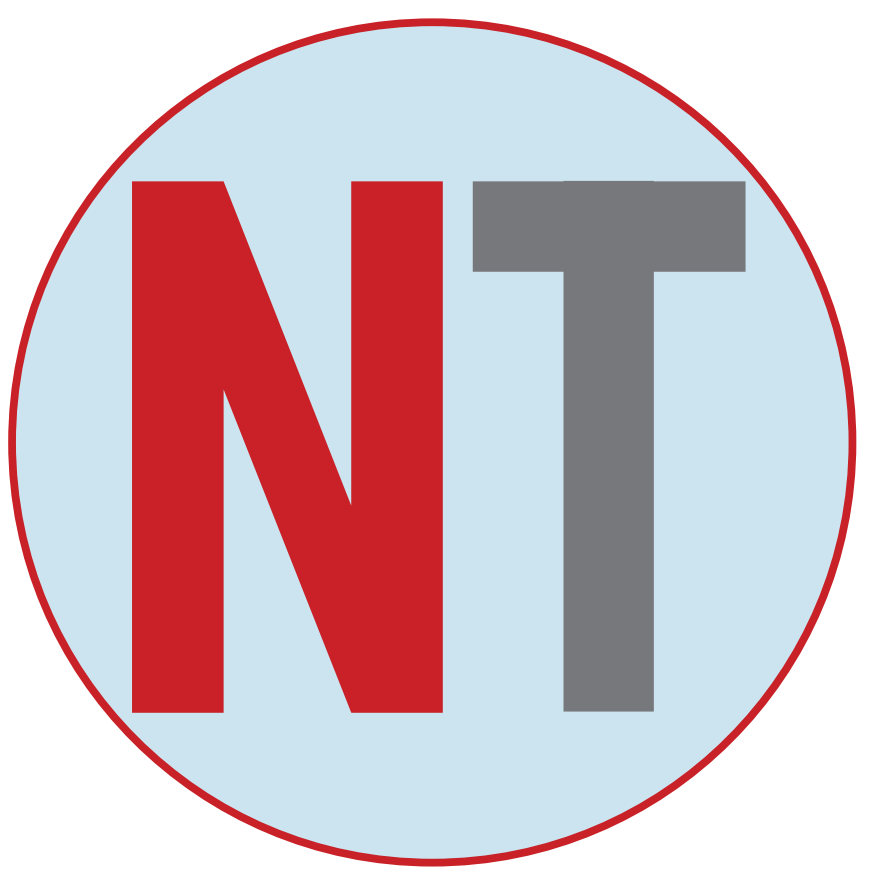

